\title{
One-dimensional metallic edge states in MoS2
}

Bollinger, Mikkel; Lauritsen, J.V.; Jacobsen, Karsten Wedel; Nørskov, Jens Kehlet; Helveg, S.; Besenbacher, Flemming

Published in:

Physical Review Letters

Link to article, DOI:

10.1103/PhysRevLett.87.196803

Publication date:

2001

Document Version

Publisher's PDF, also known as Version of record

Link back to DTU Orbit

Citation (APA):

Bollinger, M., Lauritsen, J. V., Jacobsen, K. W., Nørskov, J. K., Helveg, S., \& Besenbacher, F. (2001). Onedimensional metallic edge states in MoS2. Physical Review Letters, 87(19), 196803.

https://doi.org/10.1103/PhysRevLett.87.196803

\section{General rights}

Copyright and moral rights for the publications made accessible in the public portal are retained by the authors and/or other copyright owners and it is a condition of accessing publications that users recognise and abide by the legal requirements associated with these rights.

- Users may download and print one copy of any publication from the public portal for the purpose of private study or research.

- You may not further distribute the material or use it for any profit-making activity or commercial gain

- You may freely distribute the URL identifying the publication in the public portal

If you believe that this document breaches copyright please contact us providing details, and we will remove access to the work immediately and investigate your claim 


\title{
One-Dimensional Metallic Edge States in $\mathrm{MoS}_{2}$
}

\author{
M. V. Bollinger, ${ }^{1}$ J. V. Lauritsen, ${ }^{2}$ K. W. Jacobsen, ${ }^{1}$ J. K. Nørskov, ${ }^{1}$ S. Helveg, ${ }^{2}$ and F. Besenbacher ${ }^{2}$ \\ ${ }^{1}$ Center for Atomic-scale Materials Physics, Department of Physics, Technical University of Denmark, \\ DK-2800 Kongens Lyngby, Denmark \\ ${ }^{2}$ Center for Atomic-scale Materials Physics, Institute of Physics and Astronomy, University of Aarhus, DK-8000 Århus, Denmark \\ (Received 23 May 2001; published 18 October 2001)
}

\begin{abstract}
By the use of density functional calculations it is shown that the edges of a two-dimensional slab of insulating $\mathrm{MoS}_{2}$ exhibit several metallic states. These edge states can be viewed as one-dimensional conducting wires, and we show that they can be observed directly using scanning tunneling microscopy for single-layer $\mathrm{MoS}_{2}$ nanoparticles grown on a support.
\end{abstract}

DOI: $10.1103 /$ PhysRevLett.87.196803

When a bulk insulator is terminated by a surface, it is well known that localized two-dimensional metallic surface states may appear [1]. In the present Letter we address the question whether a two-dimensional insulating material may also show localized metallic states at the edges. Such edge states would then be a realization of a onedimensional metallic wire. The possibility of finding such edge states has previously been discussed in connection with graphene structures [2,3], but to our knowledge no experimental evidence has yet been reported. Here we focus on single-layer $\mathrm{MoS}_{2}$ nanostructures. Such structures can be grown experimentally on a reconstructed $\mathrm{Au}(111)$ substrate and studied using scanning tunneling microscopy (STM) [4]. It is found that the $\mathrm{MoS}_{2}$ nanoclusters show a brim of very high conductance extending all the way around the edge; see Fig. 1.

If the yellow brim can be associated with metallic edge states, it means that we are observing in Fig. 1 a set of nanosized, metallic, closed wires.

Using density functional theory (DFT) calculations we show in the following that the edges of single-layer $\mathrm{MoS}_{2}$ have up to two localized metallic states. These states are not associated with dangling bonds but reflect subtle changes in the electronic structure close to the edges. We also show using the Tersoff-Hamann formalism [5] that simulated STM images have a bright structure at the edge similar to the one we have observed experimentally for $\mathrm{MoS}_{2}$ clusters on $\mathrm{Au}$ and that the bright structure is associated with one of the edge states. The STM experiments can therefore be taken as direct observations of onedimensional, metallic edge states.

$\mathrm{MoS}_{2}$ is a layered compound consisting of S-Mo-S sandwiches. We consider a single layer and study the edges in a model system consisting of a stripe of $\mathrm{MoS}_{2}$ repeated in a supercell geometry; see Fig. 2.

The stripe is terminated by two kinds of edges, the sulfur-terminated (1010) edge and the molybdenumterminated $(10 \overline{1} 0)$ edge. In this Letter we focus on the Mo edge with adsorbed $\mathrm{S}$ dimers. Based on our calculated energetics [6,7] this is the most likely equilibrium edge structure in excess of $\mathrm{H}_{2} \mathrm{~S}$, and it is in addition the structure where the calculated STM picture most closely resembles
PACS numbers: 73.20.At, 61.46.+w, 61.48.+c, 68.37.Ef

that of the experimental structure in Fig. 1. There are a number of other edge structures with $\mathrm{S}$ monomers, with $\mathrm{H}$ adsorbed, or even the opposite (sulfur-terminated) edge that are close in energy, and most (but not all) of these structures show metallic edge states. The existence of the metallic edge states does therefore not depend crucially on the studied structure. This issue will be addressed in more detail elsewhere [7].

The details of the DFT calculations are as follows [8-11]: The Kohn-Sham (KS) orbitals are expanded in plane waves with kinetic energies below $25 \mathrm{Ry}$ and the Brillouin zone is sampled in a one-dimensional grid with 12 special $\boldsymbol{k}$ points [12] in the $k_{x}$ direction. The ionic cores are replaced by ultrasoft pseudopotentials [13], and to treat exchange and correlation the PW91 functional is used [14]. First, the one-electron KS equation is solved by iterative diagonalization and the resulting KS eigenstates are populated according to the Fermi distribution. Then the Pulay mixing of densities is applied and the entire procedure is repeated until self-consistency is obtained. The total energies are subsequently extrapolated to zero temperature. In all calculations the atomic structure is optimized by allowing each atom to relax according to the calculated forces.
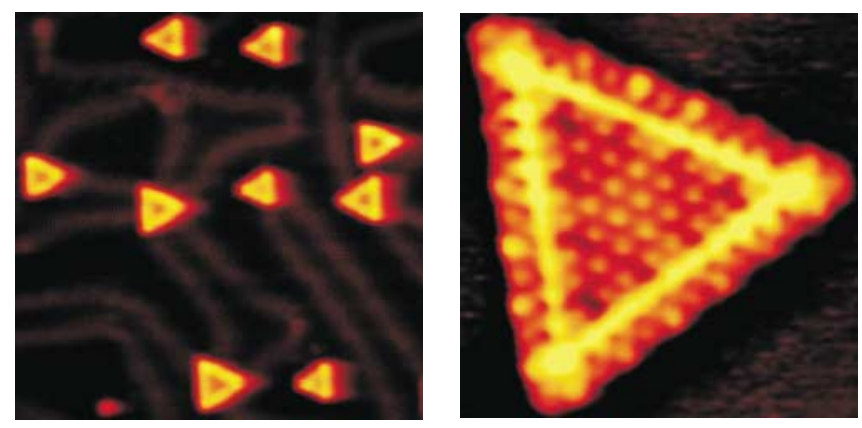

FIG. 1 (color). Left: STM image $\left(278 \times 282 \AA^{2}\right)$ showing triangular $\mathrm{MoS}_{2}$ nanoclusters dispersed on a reconstructed $\mathrm{Au}(111)$ surface. Right: Single $\mathrm{MoS}_{2}$ nanocluster $\left(48 \times 53 \AA^{2}\right.$; $\left.V_{\mathrm{t}}=5.3 \mathrm{mV} ; I_{\mathrm{t}}=1.28 \mathrm{nA}\right)$. The triangularly shaped singlelayer $\mathrm{MoS}_{2}$ nanoclusters were synthesized by depositing Mo on a reconstructed $\mathrm{Au}(111)$ surface in an $\mathrm{H}_{2} \mathrm{~S}$ atmosphere [4]. 

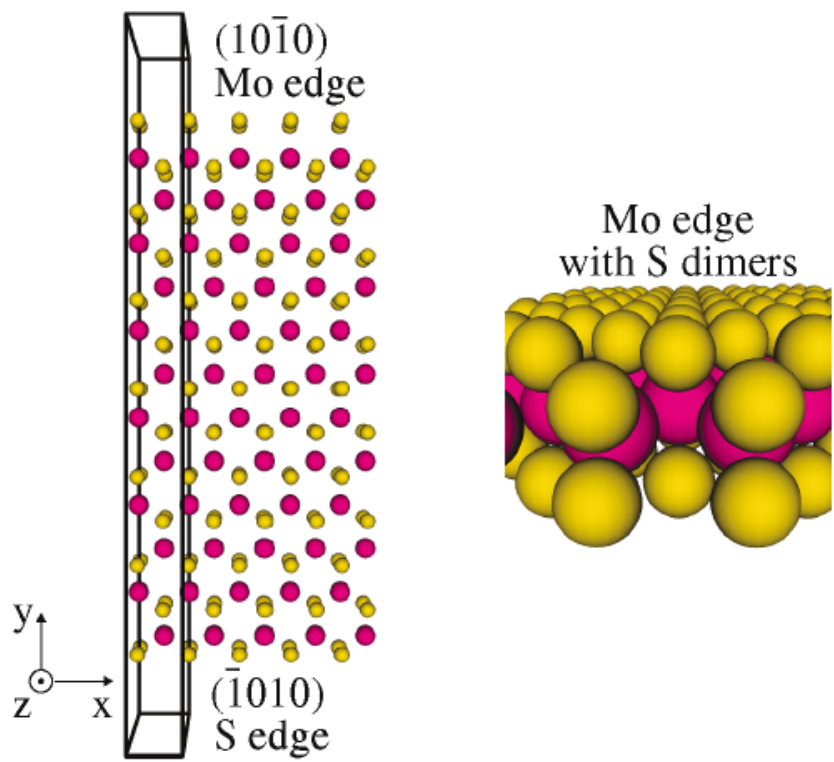

FIG. 2 (color). Model system of the single-layer $\mathrm{MoS}_{2}$ stripe with $(1,12)$ rows of Mo atoms in the $(x, y)$ direction. The yellow spheres represent $\mathrm{S}$ atoms, whereas the magenta spheres correspond to Mo atoms. The stripes are separated by $8.9 \AA$ of vacuum between the $\mathrm{MoS}_{2}$ planes and $8.8 \AA$ of vacuum in the direction perpendicular to edges. Left: the surface of the stripe is shown where the outline of the supercell is indicated farthest to the left. Right: the side of the Mo edge with S dimers is shown slightly tilted.

It is well known that bulk $\mathrm{MoS}_{2}$ is a semiconductor. The experimental band gap is $1.23 \mathrm{eV}$ [15], whereas the calculated value has been reported to be $0.77 \mathrm{eV}$ [16]. As indicated in Fig. 3 this is also the result of our DFT calculations for a single-layer $\mathrm{MoS}_{2}$ slab which we find to have a direct band gap of $1.64 \mathrm{eV}$.

For the $\mathrm{MoS}_{2}$ stripes which have edges included, the band structure has changed significantly. In Fig. 3 it is seen that the $\mathrm{MoS}_{2}$ stripe has three bands crossing the Fermi level, implying the existence of three onedimensional metallic states, labeled I, II, and III, respectively. Direct inspection of the corresponding KS wave functions reveals that III is localized at the S edge, whereas states I and II are associated with the Mo edge. The two metallic wave functions at the Mo edge are shown in Fig. 3. Edge state I has a Fermi wave vector of $k_{\mathrm{F}} \simeq 0.39 \AA^{-1}$ and is seen to be almost completely localized at the $\mathrm{S}$ dimers. It is a superposition of $p_{\mathrm{x}}$ orbitals extending in two parallel chains along the edge. Edge state II is seen to extend over the first three rows having $k_{\mathrm{F}} \simeq 0.67 \AA^{-1}$. The nature of this state is somewhat more complicated than for I. It is primarily constituted by two bonds: (1) the $d-d$ bond between the first row Mo atoms and (2) the $p$ - $d$ bond between the two second row $\mathrm{S}$ atoms and the Mo atom behind.

We now turn to the question whether the metallic edge states give rise to the striking yellow brim observed in our STM pictures of $\mathrm{MoS}_{2}$ nanoclusters seen in Fig. 1 [4]. (a)
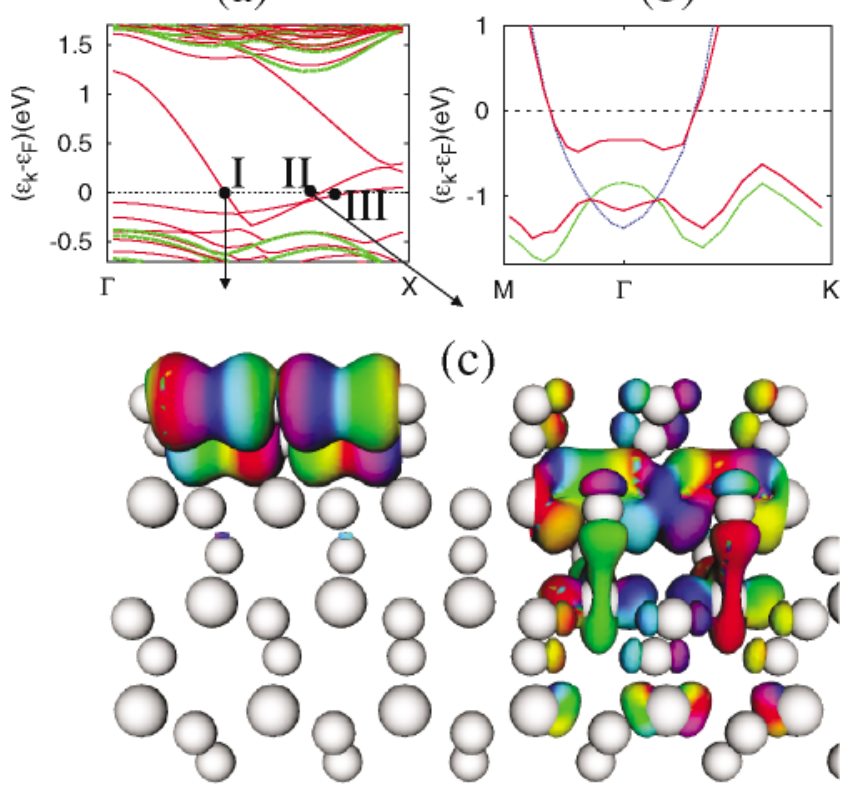

(c)

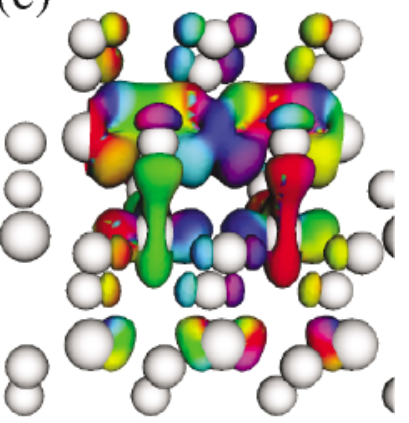

FIG. 3 (color). (a) One-dimensional energy bands around the Fermi level of a $\mathrm{MoS}_{2}$ stripe (red lines) and a $\mathrm{MoS}_{2}$ slab of the same size (green lines). The band gap of an isolated $\mathrm{MoS}_{2}$ system is clearly seen. (b) Selected energy bands of a single-layer $\mathrm{MoS}_{2}$ slab (green line) and a $\mathrm{Au}(111)$ surface (blue line). In red we show the corresponding energy bands of $\mathrm{MoS}_{2}$ on $\mathrm{Au}(111)$, and the avoided crossings leading to a slight metallic character of the $\mathrm{MoS}_{2}$ states at the Fermi level is seen. (c) Contours of the KS wave functions corresponding to the metallic states at the Mo edge. The contours are colored according to the phase of the wave functions and the rapid oscillations are due to the factor, $\exp \left(i \boldsymbol{k}_{\mathrm{F}} \cdot \boldsymbol{r}\right)$. The left side (right side), denoted I (II), corresponds to $k_{\mathrm{F}} \simeq 0.39 \AA^{-1}\left(k_{\mathrm{F}} \simeq 0.67 \AA^{-1}\right)$ as indicated by the arrows.

The nanoclusters are synthesized under ultrahigh vacuum conditions using the reconstructed $\mathrm{Au}(111)$ surface to disperse $\mathrm{MoS}_{2}$ into triangular nanocrystals with a uniform size distribution. The atomic-scale structure of clusters is explored by a homebuilt STM operated in the constantcurrent mode. Further details are described in Ref. [4]

We have simulated an STM image within the TersoffHamann model [5]. According to this model a constantcurrent STM image simply reflects an isosurface of the local density of states (LDOS). Because of the discrete sampling of the Brillouin zone this quantity is calculated by replacing each energy level with a Gaussian of finite width. The LDOS contour value is fixed by the experimental data: An STM image is simulated for a single-layer $\mathrm{MoS}_{2}$ slab on $\mathrm{Au}(111)$ [17]. The contour value is then determined by matching the experimental corrugation measured along the close-packed row of $\mathrm{S}$ atoms in the interior of a nanocrystal to the corresponding corrugation of the simulated image. In this way a value of LDOS $=8.3 \times 10^{-6}\left(\AA^{3} \mathrm{eV}\right)^{-1}$ is obtained. This corresponds to an average height of $2.72 \AA$ above the top $\mathrm{S}$ layer. We also note that, in agreement with previous studies, the simulated image shows the $\mathrm{S}$ atoms 
as protrusions whereas the depressions are associated with Mo atoms [16,18].

The simulated STM image of the Mo edge is shown in Fig. 4. It reproduces several important features of the experimental image. In particular, we clearly see the yellow brim behind the $\mathrm{S}$ dimers. Comparing with edge state I the brim is seen to be due to the $p$ orbital originating from the $p$ - $d$ bond between $\mathrm{S}$ and Mo. We also note that the protrusions in the row of the $\mathrm{S}$ dimers at the edge are not associated with the $S$ atoms but rather reflect the interstitial region between the $\mathrm{S}$ dimers. This is again in agreement with the experimental observation. The behavior can be attributed to edge state I since the constituent $p_{\mathrm{x}}$ orbitals have a node on each $\mathrm{S}$ atom.

The one-dimensional metallic edge states could be candidates to form a Peierls system resulting in the opening of an energy gap at the Fermi energy [19]. Even a small gap would, of course, destroy the conducting properties of the edge states at low bias. The STM images do, in fact, exhibit a variation with a period of two at the Mo edge, and this could be taken as support for the occurrence of an atomic rearrangement due to a Peierls instability. However, increasing the size of the unit cell to include two atomic rows, thus allowing for a periodicity of two, does not show any change in either the atomic or the electronic structure. The result is not surprising since the values of $\boldsymbol{k}_{\mathrm{F}}$ for both metallic states differ from half filling. Indeed, it turns out that given the values $\boldsymbol{k}_{\mathrm{F}}=0.39$ and $0.67 \AA^{-1}$, it is not possible for either of the wave vectors to increase the periodicity of the system such that they coincide

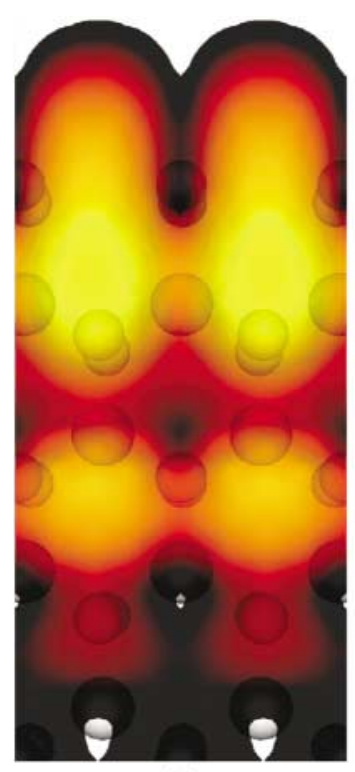

(a)

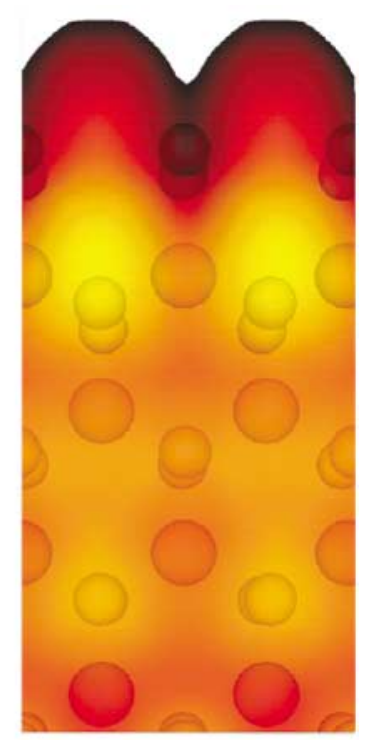

(b)
FIG. 4 (color). Simulated STM image for the model system with (a) and without (b) an underlying $\mathrm{Au}(111)$ substrate. For clarity the $\mathrm{Au}$ atoms have not been retained in the $\mathrm{MoS}_{2} / \mathrm{Au}(111)$ STM image. The color scale is black $\rightarrow$ red $\rightarrow$ yellow and corresponds to a corrugation of $1.5 \AA$. with the boundary of the first Brillouin zone for a reasonably small unit cell. The DFT calculations therefore suggest that the metallic edge states are stable against atomic rearrangements.

This leaves us with the question why the STM images show a period two modulation at the edge. We suggest that it may be due to the finite size of the $\mathrm{MoS}_{2}$ nanoclusters. The corners of the nanoclusters may, for example, induce atomic relaxations along the edges since the calculations show that the horizontal $\mathrm{S}$ dimer potential is very weak. (A displacement of $0.4 \AA$ changes only the energy on the order of $10 \mathrm{meV}$.) The finite size could also give rise to standing wave patterns at the Fermi energy. Calculations on small triangular $\mathrm{MoS}_{2}$ clusters do, in fact, show indications of a period of two at the edges [7]. Given the complexity of the system other explanations can therefore not be ruled out. In particular, if the experimental values of $\boldsymbol{k}_{\mathrm{F}}$ differ from the corresponding calculated values, the possibility of a broken-symmetry ground state is evident.

The $\mathrm{MoS}_{2}$ clusters shown in Fig. 1 are deposited on an $\mathrm{Au}$ surface and the question arises whether the edge states and the imaging of these states are strongly influenced by the presence of the substrate. Clearly the substrate must be important for the imaging of the interior of the clusters: $\mathrm{MoS}_{2}$ is an insulator with a band gap much larger than the bias applied in the STM experiment. Without the interaction with the substrate imaging of the interior would not be possible except for effects caused by defects in the $\mathrm{MoS}_{2}$. This is also implied by the calculated STM image of the $\mathrm{MoS}_{2}$ stripe shown in Fig. 4. In order to investigate the influence of the Au substrate we have carried out calculations for the $\mathrm{MoS}_{2}$ stripe with an underlying $\mathrm{Au}(111)$ surface. The simulated STM image is shown in Fig. 4, and here it is seen that the imaging of the edge remains essentially unchanged. This in turn indicates that the underlying electronic structure of the metallic edge states is not significantly perturbed. We note that the calculated height of the yellow brim relative to the height of the basal plane $(0.39 \AA)$ is in agreement with the measured value $(0.4 \pm 0.1 \AA)$.

It should be mentioned that $\mathrm{MoS}_{2}$ triangles of finite size will, in principle, have a discrete electronic excitation spectrum. The level spacing can be estimated as $W / N_{\text {sites }}$, where $W$ is the bandwidth. The metallic edge states in Fig. 3 have a bandwidth of the order $1.0 \mathrm{eV}$, and for a triangle of the size shown in Fig. 1 we thus obtain $1.0 \mathrm{eV} / 40=25 \mathrm{meV}$. Hence, even at room temperature electronic states above the Fermi level are available for conduction at small bias voltages.

We have shown that two one-dimensional metallic channels exist intrinsically in single-layer $\mathrm{MoS}_{2}$, and we have shown that these states can be directly observed experimentally using STM. One-dimensional metallic wires have been studied extensively during the last two decades using semiconductor heterostructures [20]. In the mesoscopic regime, that is, on the length scale of $\mu \mathrm{m}$ for 
these systems, interference of the conduction electrons becomes important and results in pure quantum phenomena such as the Aharanov-Bohm effect, persistent currents, and weak localization. The closed one-dimensional metallic wires formed by the single-layer $\mathrm{MoS}_{2}$ clusters may provide a template for studies of quantum effects on a length scale 3 orders of magnitude smaller than what can be achieved using conventional semiconductor techniques.

The present work was in part financed by The Danish Research Councils through Grant No. 9501775. The Center for Atomic-scale Materials Physics is sponsored by the Danish National Research Foundation.

[1] A. Zangwill, Physics at Surfaces (Cambridge University Press, Cambridge, 1988).

[2] K. Nakada, M. Fujita, G. Dresselhaus, and M. S. Dresselhaus, Phys. Rev. B 54, 17954 (1996).

[3] Y. Miyamoto, K. Nakada, and M. Fujita, Phys. Rev. B 59, 9858 (1999).

[4] S. Helveg, J. V. Lauritsen, E. Lægsgaard, I. Stensgaard, J. K. Nørskov, B. S. Clausen, H. Topsøe, and F. Besenbacher, Phys. Rev. Lett. 84, 951 (2000).

[5] J. Tersoff and D. R. Hamann, Phys. Rev. B 31, 805 (1985).

[6] L. S. Byskov, J. K. Nørskov, B. S. Clausen, and H. Topsøe, J. Catal. 187, 109 (1999).

[7] M. V. Bollinger, K. W. Jacobsen, J. K. Nørskov, J. V. Lauritsen, B.S. Clausen, H. Topsøe, S. Helveg, and F. Besenbacher (to be published).
[8] B. Hammer and O. H. Nielsen, in Proceedings of the Second International Workshop on Applied Parallel Computing in Physics, Chemistry and Engineering Science (PARA '95), Lyngby, Denmark, 1995, edited by J. J. Dongarra, K. Madsen, and J. Wasniewski, Springer Lecture Notes in Computer Science Vol. 1041 (Springer, Berlin, New York, 1996).

[9] B. Hammer, L. B. Hansen, and J. K. Nørskov, Phys. Rev. B 59, 7413 (1999).

[10] G. Kresse and J. Furthmüller, Comput. Mater. Sci. 6, 15 (1996).

[11] The calculations were performed with the Dacapo pseudopotential code, http://www.fysik.dtu.dk/campos

[12] H. J. Monkhorst and J. D. Pack, Phys. Rev. B 13, 5188 (1976).

[13] D. Vanderbilt, Phys. Rev. B 41, 7892 (1990).

[14] J. P. Perdew, J. A. Chevary, S. H. Vosko, K. A. Jackson, M. R. Pederson, D. J. Singh, and C. Fiolhais, Phys. Rev. B 46, 6671 (1992).

[15] K. K. Park and B. A. Parkinson, J. Phys. Chem. 86, 463 (1982).

[16] K. Kobayashi and J. Yamauchi, Phys. Rev. B 51, 17085 (1995).

[17] The Au(111) surface is modeled as a rigid two layer slab assuming epitaxial growth of $\mathrm{MoS}_{2}$.

[18] A. Altibelli, C. Joachim, and P. Sautet, Surf. Sci. 367, 209 (1996).

[19] C. Kittel, Introduction to Solid State Physics (Wiley, New York, 1996).

[20] Y. Imry, Introduction to Mesoscopic Physics (Oxford University Press, New York, 1997). 